\title{
Expectation and experience: Passenger acceptance of autonomous public transportation vehicles
}

\author{
Grace Eden ${ }^{1}$, Benjamin Nanchen ${ }^{1}$, Randolf Ramseyer ${ }^{1}$, Florian Evéquoz ${ }^{1,2}$ \\ ${ }^{1}$ University of Applied Sciences of Western Switzerland, HES-SO, 3960 Sierre, Switzerland \\ \{grace.eden, benjamin.nanchen, randolf.ramseyer, \\ florian.evequoz @ hevs.ch \\ ${ }^{2}$ University of Fribourg, 1700 Fribourg, Switzerland \\ \{florian.evequoz\} @unifr.ch
}

\begin{abstract}
Passenger acceptance is a key factor for the successful integration, uptake and use of autonomous vehicles (AVs) in the domain of public transportation. Especially knowing opinions and attitudes around safety, comfort and convenience. We discuss a pilot study conducted as part of a larger research project where AVs are being tested to transport members of the general public on a specified route with designated stops. We present preliminary findings of fieldwork conducted where people were asked their opinions and attitudes both before and after riding on an AV shuttle as a passenger for the first time. This allows us to compare user expectation beforehand with actual experience afterwards.
\end{abstract}

Keywords. Autonomous vehicles, Public transportation, Human-Machine Interaction, Passenger acceptance, Fieldwork.

\section{$1 \quad$ Introduction and background}

Autonomous passenger vehicles are being piloted across the globe to assess their technically and operationally feasibility. In June 2016, PostBus, the primary public bus transportation provider in Switzerland and the Mobility Lab Sion-Valais joined together for a pilot study, the Sion Smart Shuttle. The project began in a cordoned-off private area from December 2015 to Spring 2016. However, once government approval was granted in June 2016, the testing was moved on to public roadways and dual use vehicle/pedestrian areas. This phase of the pilot will run until October 2017.

In this project commercial and academic partners collaborate to develop novel mobility services. In parallel with technical development, understanding customer behaviour and acceptance of the AVs and in particular passenger reactions to riding on the shuttle is also being investigated. This paper presents preliminary findings of a pilot case study as part of a larger project where we also investigate how other road users inter- 
pret AV behaviour [1]. For this pilot study we conducted interviews with passengers both before and after riding on the shuttle to provide us with an opportunity to compare user expectation beforehand with actual experience afterwards.

\section{Passenger opinions of $\mathrm{AV}$ public transport}

The Smart Shuttle is the first pilot project of AVs on public roads in Switzerland and operates on a route of 1.5 kilometers in the Old Town district of Sion. After months of refining and stabilizing the technical aspects of the AVs mapping and sensor operations to ensure safety, the first passenger acceptance pilot study commenced over a one-month period from November-December 2016 with an aim to understand passenger opinions of AV public transportation.

The AV Shuttle can hold up to 11 people with an attendant on board monitoring, and at times taking over, its operation. We conducted nine fieldwork sessions with participants who agreed to be interviewed and to take a ride on the AV shuttle. The study included 17 passengers: 3 individuals, 4 couples, and 2 groups of three. In addition, the sessions were also video-recorded using two mounted action cameras: one with an interior view of passengers and the other with an exterior view of the road ahead (Fig.1).

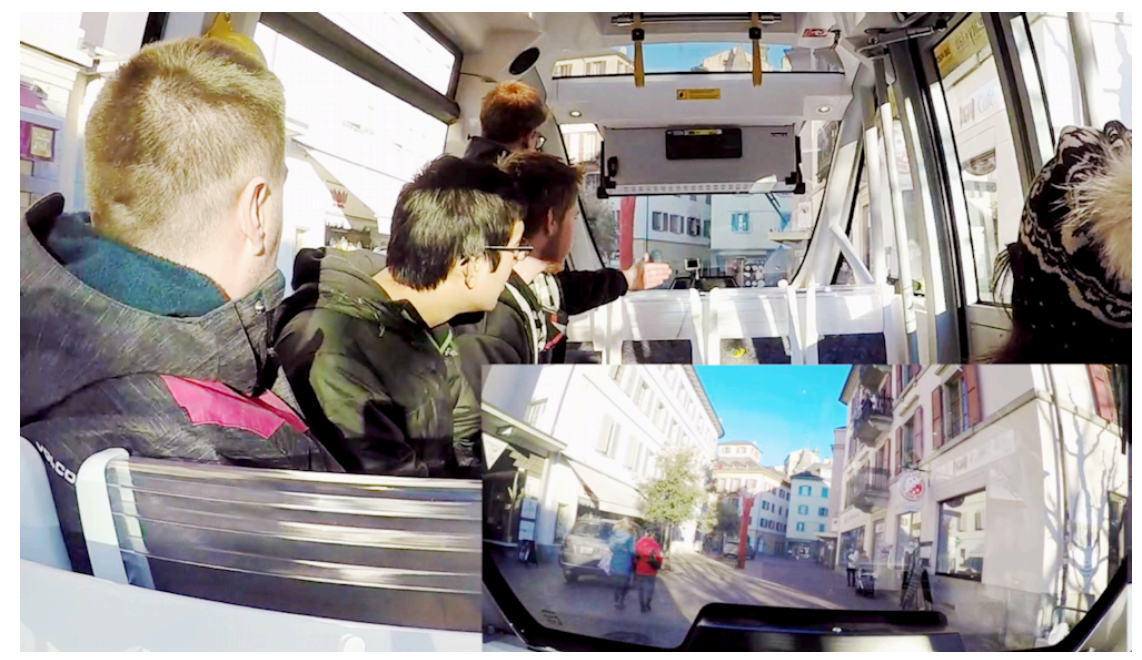

Fig. 1. Two mounted action cameras record passenger and road activity simultaneously.

The fieldwork had three components. First, participants were briefly interviewed before riding the shuttle and asked to describe their initial expectations including perceptions of safety, comfort and any other feelings and opinions. Second, we conducted video-based observation [2] of participants' actual journey on the shuttle. Third, after riding on the shuttle we conducted a post-ride interview with the same questions to compare if and how their opinions may have changed. The interview data was ana- 
lyzed using qualitative methods informed by thematic analysis [3]. The preliminary findings discussed here are taken from the pre and post ride interviews with a focus on passengers' opinions related to the AV's safety, comfort and convenience.

\subsection{Opinions about safety}

Before riding on the AV shuttle 4 participants expressed safety concerns because of news reports of an accident in September 2016 [4]. The shuttle hit the fender of a stationary van while in autonomous mode. Fortunately, there were no injuries as a result of the collision although the fact that it happened made some people uneasy. Others expressed concerns related to the reliability of braking and turning. Finally, the technology in general was brought into question as one participant noted: "I don't trust it much because something could go wrong with the IT system. You go on it but you don't trust it 100\%". Even so, the 13 remaining participants said they had no concerns before riding on the shuttle primarily because of its very slow speed (maximum of 20km). Also, many had experience with other types of driverless transportation such as driverless metro trains at airports. Even though these are on guided tracks some people still felt that this experience was in some way similar.

After riding the shuttle all participants who had safety concerns beforehand no longer had them afterwards. These overall positive opinions were encouraging. Although, many passengers commented that seatbelts are necessary especially because the AV lurches forward when it makes sudden hard stops. It frequently does this when it detects an 'obstacle' such as a pedestrian, car or bicycle passing close by. Many participants said they were impressed with the automated navigation, including its steering ability through narrow spaces. Although participants responses were positive, there is an important caveat: most agreed that their perception of safety might change (safety concerns would increase) if it was a large-sized bus with no attendants on board travelling on an actual route at regular speed.

\subsection{Satisfaction with comfort and convenience}

Another key factor to passenger acceptance is comfort and convenience. The shuttle's large panoramic windows were received positively because of the wide view it provides to the outside world. However, most participants said that the comfort of the seats could be improved because they were too hard and that seatbelts are needed to prevent people from lurching forward during sudden hard stops. Additionally, 4 participants commented that the noise from the hydraulic compressor was too loud. Many participants commented on its small size saying that people are sitting too close together and that it would need to be larger to accommodate luggage and shopping bags.

Regarding convenience, all participants said that the current route was not practical because it is in a largely pedestrian area of the old town that people prefer to walk through. Rather, many said that they would like to use it for more practical journeys 
such as from the train station, the local airport, or the park and ride. This feedback indicates that there is acceptance of the AVs as a potentially useful addition to the public transportation network for smaller routes that may not be served by large buses. However, many participants felt that the hours of operation would need to be extended (it currently operates from 1-6pm). Others wanted to become more involved in the project. For instance, by adding a social media component that would allow them to share their experience online with their friends and family. Also, some suggest that an information sheet be available explaining the technology and how the AV operates.

\section{Discussion and further work}

This pilot study provides valuable insights of passenger opinions related to safety, comfort and convenience (Table 1) with each of these dimensions having implications for scalability. For instance, many participants said that the speed is slow enough to feel safe but too slow for going to work in the morning.

\begin{tabular}{|l|l|l|}
\hline Safety & Comfort & Convenience \\
\hline Braking & Windows & Route availability \\
\hline Turning & Seats & Hours of operation \\
\hline Speed & Hydraulic compressor noise & Speed \\
\hline Human co-driver & Interior size & Luggage space \\
\hline
\end{tabular}

Table 1. Dimension of safety, comfort, and convenience for AV shuttle passengers.

In further work we will conduct participatory design workshops with passengers to investigate possible future interactions with $\mathrm{AV}$ public transportation. These will examine designs for maintaining passenger trust. For instance, providing greater transparency of the $\mathrm{AV}$ decision-making process including the choices it makes around obstacle detection, braking and steering. In these workshops we will co-design and prototype a dedicated interface to monitor these activities. Lessons learned include the value of conducting fieldwork in three phases: pre and post ride interviews (for comparisons before and after) and video (for investigating communication between the AVs and other road users). In each of these areas, our aim is to inform the design of new communication and interaction mechanisms between AVs and humans.

\section{$4 \quad$ References}

1. Grace Eden, Benjamin Nanchen, Randolf Ramseyer, and Florian Evéquoz. 2017. On the Road with an Autonomous Passenger Shuttle: Integration in Public Spaces. In Proc. CHI'17, Denver CO, New York: ACM Press, pp. 1569-1576.

2. Christian Heath, Jon Hindmarsh, and Paul Luff. 2010. Video in Qualitative Research: Analysing social interaction in everyday life. London: Sage.

3. Greg Guest, Kathleen M. MacQueen, and Emily E. Namey. (2012). Applied thematic analysis. Thousand Oaks, CA: SAGE Publications.

4. https://www.postauto.ch/en/news/smartshuttle-testing-sion-resumes 